UMTG-177

\title{
Direct Calculation of the Boundary $S$ Matrix for the Open Heisenberg Chain
}

M. T. Grisaru†, Luca Mezincescu*, and Rafael I. Nepomechie*

\begin{abstract}
We calculate the boundary $S$ matrix for the open antiferromagnetic spin $1 / 2$ isotropic Heisenberg chain with boundary magnetic fields. Our approach, which starts from the model's Bethe Ansatz solution, is an extension of the Korepin-Andrei-Destri method. Our result agrees with the boundary $S$ matrix for the boundary sine-Gordon model with $\beta^{2} \rightarrow$ $8 \pi$ and with "fixed" boundary conditions.
\end{abstract}

$\dagger$ Physics Department, Brandeis University, Waltham, MA 02254

* Department of Physics, University of Miami, Coral Gables, FL 33124 


\section{Introduction}

In a $1+1$-dimensional theory with factorized scattering, the two-particle $S$ matrix, which depends on the rapidity difference $\lambda$ of the two particles and which we denote here by $R(\lambda)$, is constrained to satisfy the factorization (Yang-Baxter) equation

$$
R_{12}\left(\lambda-\lambda^{\prime}\right) R_{13}(\lambda) R_{23}\left(\lambda^{\prime}\right)=R_{23}\left(\lambda^{\prime}\right) R_{13}(\lambda) R_{12}\left(\lambda-\lambda^{\prime}\right)
$$

For particles in an $n$-dimensional representation of some internal symmetry group, $R(\lambda)$ is a matrix acting in the tensor product space $C^{n} \otimes C^{n}$. Moreover, $R_{12}, R_{13}$, and $R_{23}$ are matrices acting in $C^{n} \otimes C^{n} \otimes C^{n}$, with $R_{12}=R \otimes 1, R_{23}=1 \otimes R$, etc. (See, e.g., Refs. 1, 2 and references therein.)

For a system with a boundary, the quantum-mechanical scattering of a particle with the boundary is described by a so-called boundary $S$ matrix, which we denote here by $K(\lambda, \xi)$. This is a matrix acting in the space $C^{n}$, which may depend on one or more parameters, which we denote collectively by $\xi$, that characterize the interaction at the boundary. The condition that boundary scattering be compatible with factorization is ${ }^{3-5}$

$$
R_{12}\left(\lambda-\lambda^{\prime}\right) K_{1}(\lambda, \xi) R_{21}\left(\lambda+\lambda^{\prime}\right) K_{2}\left(\lambda^{\prime}, \xi\right)=K_{2}\left(\lambda^{\prime}, \xi\right) R_{12}\left(\lambda+\lambda^{\prime}\right) K_{1}(\lambda, \xi) R_{21}\left(\lambda-\lambda^{\prime}\right)
$$

where

$$
R_{21}(\lambda) \equiv \mathcal{P}_{12} R_{12}(\lambda) \mathcal{P}_{12}
$$

and $\mathcal{P}_{12}$ is the permutation matrix in $C^{n} \otimes C^{n}$,

$$
\mathcal{P}_{12}(x \otimes y)=y \otimes x \quad \text { for } \quad x, y \in C^{n} .
$$

Furthermore, we use the notation $K_{1}=K \otimes 1, K_{2}=1 \otimes K$.

Given $R(\lambda)$, Eq. (1.2) determines the general form of $K(\lambda, \xi)$ up to a scalar factor, which is a function of $\lambda$ and $\xi$. Such boundary $S$ matrices have been used to construct 
and to obtain the Bethe-Ansatz solution for open integrable quantum spin chains ${ }^{4-14}$. For these applications, what is important is the matrix structure of $K(\lambda, \xi)$; the scalar factor plays no significant role. Further applications of boundary $S$ matrices are reviewed in Ref. 15.

Recently, there has been interest ${ }^{16-20}$ in determining the boundary $S$ matrices for physical excitations of integrable theories. This requires determining in particular the scalar factor of the boundary $S$ matrix, which contains information about possible boundary bound states, etc.

One way to determine the scalar factor is the "bootstrap" approach: in addition to Eq. (1.2), one imposes on the boundary $S$ matrix the constraints of unitarity, boundary cross-unitarity ${ }^{18}$, and boundary bootstrap ${ }^{16,18}$ (which in the context of spin chains is known as "fusion" of boundary $S$ matrices 6 ). These conditions determine the scalar factor up to a CDD-type of ambiguity. Evidently, this procedure is a generalization of the bootstrap approach for determining bulk $S$ matrices ${ }^{1}$. Ghoshal and Zamolodchikov ${ }^{18}$ have determined in this manner the boundary $S$ matrix for the boundary sine-Gordon model.

In this paper, we consider an alternative approach: namely, to determine the boundary $S$ matrix directly from the "microscopic" theory for the excitations - i.e., from the Bethe Ansatz equations. In particular, we calculate in this way the boundary $S$ matrix for the physical excitations ("spinons") of the open antiferromagnetic spin $1 / 2$ Heisenberg chain with boundary magnetic fields. The Hamiltonian of the chain is given by

$$
\mathcal{H}=\frac{1}{4}\left\{\sum_{n=1}^{N-1} \vec{\sigma}_{n} \cdot \vec{\sigma}_{n+1}+\frac{1}{\xi_{-}} \sigma_{1}^{z}+\frac{1}{\xi_{+}} \sigma_{N}^{z}\right\},
$$

where $\vec{\sigma}$ are the usual Pauli matrices, and the real parameters $\xi_{ \pm}$correspond to boundary magnetic fields. Although the bulk terms are $S U(2)$ invariant, the boundary terms break this symmetry down to $U(1)$, which is generated by

$$
S^{z}=\frac{1}{2} \sum_{n=1}^{N} \sigma_{n}^{z}
$$


The Bethe Ansatz solution of this model is given in Refs. 4 and 21 .

Our approach is a generalization to the case of systems with boundaries of the KorepinAndrei-Destri method ${ }^{22,23}$, which was devised to calculate factorized bulk $S$ matrices for systems with periodic boundary conditions. A key ingredient is the quantization condition for a finite interval, which has recently been discussed by Fendley and Saleur ${ }^{24}$. Our result $K\left(\lambda, \xi_{ \pm}\right)$for the boundary $S$ matrix is the diagonal matrix (see Eqs. (5.19),(5.30), (5.36))

$$
K\left(\lambda, \xi_{ \pm}\right)=\alpha\left(\lambda, \xi_{ \pm}\right)\left(\begin{array}{cc}
1 & 0 \\
0 & -\frac{\lambda+i\left(\xi_{ \pm}-\frac{1}{2}\right)}{\lambda-i\left(\xi_{ \pm}-\frac{1}{2}\right)}
\end{array}\right)
$$

where the scalar factor $\alpha\left(\lambda, \xi_{ \pm}\right)$is given by

$$
\alpha\left(\lambda, \xi_{ \pm}\right)=\frac{\Gamma\left(\frac{-i \lambda}{2}+\frac{1}{4}\right)}{\Gamma\left(\frac{i \lambda}{2}+\frac{1}{4}\right)} \frac{\Gamma\left(\frac{i \lambda}{2}+1\right)}{\Gamma\left(\frac{-i \lambda}{2}+1\right)} \frac{\Gamma\left(\frac{-i \lambda}{2}+\frac{1}{4}\left(2 \xi_{ \pm}-1\right)\right)}{\Gamma\left(\frac{i \lambda}{2}+\frac{1}{4}\left(2 \xi_{ \pm}-1\right)\right)} \frac{\Gamma\left(\frac{i \lambda}{2}+\frac{1}{4}\left(2 \xi_{ \pm}+1\right)\right)}{\Gamma\left(\frac{-i \lambda}{2}+\frac{1}{4}\left(2 \xi_{ \pm}+1\right)\right)}
$$

Let us compare the above result with previous field theory results. The bulk $S$ matrix for the Heisenberg chain ${ }^{25}$ coincides with the bulk $S$ matrix for the sine-Gordon model ${ }^{1}$ in the limit $\beta^{2} \rightarrow 8 \pi$, which is the $S U(2)$-invariant point. * Therefore, we expect that the boundary $S$ matrix for the open Heisenberg chain with boundary magnetic fields (1.7), (1.8) should coincide with the boundary $S$ matrix of Ghoshal and Zamolodchikov ${ }^{18}$ for the boundary sine-Gordon model with $\beta^{2} \rightarrow 8 \pi$ and with "fixed" boundary conditions. (For "fixed" boundary conditions, the field theory and hence the boundary $S$ matrix are $U(1)$ invariant.) We have verified that the two boundary $S$ matrices indeed coincide, up to a rapidity-independent scalar factor, and with some redefinitions of variables. $\dagger$

* More precisely, the true $S$ matrices $\check{R}=\mathcal{P} R$ for the two models are related by a unitary transformation.

$\dagger$ In the notation of Ref. 18, "fixed" boundary conditions corresponds to the case $k=0$. Moreover, the isotropic limit is performed by rescaling $\xi \rightarrow \lambda \xi-\frac{\pi}{2}$ (here $\left.\lambda \equiv\left(8 \pi / \beta^{2}\right)-1\right)$, and taking the limit $\lambda \rightarrow 0$ with rapidity $u=-i \theta$ fixed. Our result for the boundary $S$ matrix is recovered by making the identifications $\theta=\pi \lambda$ and $\xi=\pi\left(\xi_{ \pm}-\frac{1}{2}\right)$. 
The bootstrap result of Ghoshal and Zamolodchikov for the boundary sine-Gordon model with "fixed" boundary conditions has been verified using the physical Bethe Ansatz approach by Fendley and Saleur ${ }^{24}$.

We now outline the contents of the paper. In Section 2, we consider the problem of quantization on a finite interval. We begin by demonstrating that even the simplest of systems can exhibit interesting boundary $S$ matrices. Indeed, we reanalyze an example which is familiar to every student of quantum mechanics - a free nonrelativistic particle but with nonstandard boundary conditions. This leads to a boundary $S$ matrix which is momentum dependent, and which has a pole which may correspond to a boundary bound state. We work out the quantization condition on a finite interval, and then discuss the generalization for factorized scattering. As already noted, this quantization condition is a key ingredient of the calculation of boundary $S$ matrices from the Bethe Ansatz. In Section 3 we analyze the Bethe Ansatz equations for the model (1.5) using the string hypothesis. In Section 4, we study the ground state. In particular, we compute the root density to order $1 / N$, and calculate the surface energy. Although these results have previously been obtained ${ }^{26}$, the calculations serve as a useful preparation for the study of excited states. Section 5 is the core of the paper. There we compute the density of roots and holes to order $1 / N$ for excited states. With the help of the quantization condition, we then determine the boundary $S$ matrix. We also perform a nontrivial consistency check on our result. There is a brief discussion in Section 6. At several points in Sections 4 and 5, we must approximate certain sums by integrals, being careful to keep terms of order $1 / N$. We derive an appropriate formula in the Appendix with the help of the Euler-Maclaurin formula. 


\section{Quantization on a finite interval}

\section{Nonrelativistic scattering}

As a warm-up exercise, we first consider a free 1-dimensional nonrelativistic particle of mass $m$ on the half-line $x \geq 0$. Usually one demands that the wavefunction $\psi(x)$ vanish at $x=0$. This is a sufficient, but by no means necessary, condition for the probability

current $j(x)=i \psi(x) * \stackrel{\leftrightarrow}{\partial}_{x} \psi(x)$ to vanish at $x=0$. We consider instead the more general (mixed Dirichlet-Neumann) boundary condition

$$
c \psi(x)+\frac{d}{d x} \psi(x)=0 \quad \text { at } x=0
$$

where $c$ is a real parameter of dimension $1 /$ length. This boundary condition also implies the vanishing of the probability current at $x=0$, and is compatible with the self-adjointness of the Hamiltonian $H=p^{2} / 2 m$. Assuming energy eigenfunctions of the plane-wave form

$$
\psi_{p}(x)=A e^{i p x}+B e^{-i p x}
$$

(we set $\hbar=1$ ), we can use the boundary condition (2.1) to eliminate $A$ in terms of $B$; and we immediately obtain

$$
\psi_{p}(x)=B\left[e^{-i p x}+\left(\frac{p+i c}{p-i c}\right) e^{i p x}\right]
$$

We conclude that the boundary $S$ matrix is given by

$$
K(p)=\frac{p+i c}{p-i c}
$$

The pole at $p=i c$ implies the existence (for $c>0$ ) of a boundary bound state with energy $E=-c^{2} / 2 m$.

We remark that for $c=0$, we have the Neumann boundary condition $\psi^{\prime}(0)=0$, and the wavefunction is

$$
\psi_{p}(x) \sim \cos p x
$$


while for $c \rightarrow \infty$, we have the Dirichlet boundary condition $\psi(0)=0$, and the wavefunction is

$$
\psi_{p}(x) \sim \sin p x
$$

Wavefunctions with such properties have appeared in quantum impurity problems. (See, e.g., Ref. 27.)

We next consider the problem of a free particle on the finite interval $-L / 2 \leq x \leq L / 2$, with mixed Dirichlet-Neumann boundary conditions at both ends:

$$
\mp c_{ \pm} \psi(x)+\frac{d}{d x} \psi(x)=0 \quad \text { at } x= \pm \frac{L}{2}
$$

Assuming again that the energy eigenfunctions are plane waves (2.2), and imposing the above boundary conditions, we see that $p$ obeys the quantization condition

$$
e^{i 2 p L} K^{-}(p) K^{+}(p)=1
$$

where the boundary $S$ matrices $K^{ \pm}$are given by

$$
K^{ \pm}(p)=\frac{p+i c_{ \pm}}{p-i c_{ \pm}}
$$

Of course, the momentum operator is not defined on a finite interval, and therefore $p$ is not a momentum eigenvalue. Nevertheless, the energy is still given by $E=p^{2} / 2 m$.

Finally, if we now turn on a reflectionless potential which is localized near $x=0$, the quantization condition $(2.8)$ is generalized to

$$
e^{i 2 p L} R(p)^{2} K^{-}(p) K^{+}(p)=1
$$

where $R(p)$ is the $S$ matrix for the potential. We shall now see that this formula has a straightforward generalization for factorized scattering. 


\section{Factorized scattering}

Consider a system of particles with factorized scattering on an interval of finite length $L$. Each particle has some rapidity $\lambda$, and in general also carries internal quantum numbers, such as spin. We denote the energy of a particle by $\varepsilon(\lambda)$, and we define $p(\lambda)$ by the expression for the momentum of a particle for the corresponding system with periodic boundary conditions. For the case of two such particles, with corresponding rapidities $\lambda_{1}$ and $\lambda_{2}$, the quantization condition reads (in the notation used in Eqs.(1.1) - (1.4))

$$
e^{i 2 p\left(\lambda_{1}\right) L} R_{12}\left(\lambda_{1}-\lambda_{2}\right) K_{1}\left(\lambda_{1}, \xi_{-}\right) R_{21}\left(\lambda_{1}+\lambda_{2}\right) K_{1}\left(\lambda_{1}, \xi_{+}\right)=1
$$

This quantization condition has recently been discussed in Ref. 24. As noted there, such a formula can be derived with the help of the Zamolodchikov-Faddeev algebra.

\section{Bethe Ansatz and string hypothesis}

The Hamiltonian $\mathcal{H}$ for the open antiferromagnetic Heisenberg chain with boundary magnetic fields is given by Eq. (1.5). The simultaneous eigenstates of $\mathcal{H}$ and $S^{z}$ have been determined by both the coordinate ${ }^{21}$ and algebraic ${ }^{4}$ Bethe Ansatz. In the latter approach, one constructs certain creation and destruction operators, $\mathcal{B}(\lambda)$ and $\mathcal{C}(\lambda)$, respectively; and the eigenstates are given by

$$
\mathcal{B}\left(\lambda_{1}\right) \mathcal{B}\left(\lambda_{2}\right) \cdots \mathcal{B}\left(\lambda_{M}\right) \omega^{+}
$$

where $\omega^{+}$is the ferromagnetic vacuum state with all spins up,

$$
\mathcal{C}(\lambda) \omega^{+}=0
$$

and $\left\{\lambda_{\alpha}\right\}$ satisfy the Bethe Ansatz (BA) equations

$$
\begin{gathered}
\left(\frac{\lambda_{\alpha}+i\left(\xi_{+}-\frac{1}{2}\right)}{\lambda_{\alpha}-i\left(\xi_{+}-\frac{1}{2}\right)}\right)\left(\frac{\lambda_{\alpha}+i\left(\xi_{-}-\frac{1}{2}\right)}{\lambda_{\alpha}-i\left(\xi_{-}-\frac{1}{2}\right)}\right)\left(\frac{\lambda_{\alpha}+\frac{i}{2}}{\lambda_{\alpha}-\frac{i}{2}}\right)^{2 N}=\prod_{\beta \neq \alpha}^{M}\left(\frac{\lambda_{\alpha}-\lambda_{\beta}+i}{\lambda_{\alpha}-\lambda_{\beta}-i}\right)\left(\frac{\lambda_{\alpha}+\lambda_{\beta}+i}{\lambda_{\alpha}+\lambda_{\beta}-i}\right) \\
\alpha=1, \cdots, M .
\end{gathered}
$$


The corresponding energy eigenvalues are given by

$$
E=-\frac{1}{2} \sum_{\alpha=1}^{M} \frac{1}{\lambda_{\alpha}^{2}+\frac{1}{4}}
$$

(plus terms that are independent of $\left\{\lambda_{\alpha}\right\}$ ), and the corresponding $S^{z}$ eigenvalues are given by

$$
S^{z}=\frac{N}{2}-M
$$

The BA equations can be written more compactly as

$$
e_{2 \xi_{+-1}}\left(\lambda_{\alpha}\right) e_{2 \xi_{--1}}\left(\lambda_{\alpha}\right) e_{1}\left(\lambda_{\alpha}\right)^{2 N+1}=-\prod_{\beta=1}^{M} e_{2}\left(\lambda_{\alpha}-\lambda_{\beta}\right) e_{2}\left(\lambda_{\alpha}+\lambda_{\beta}\right)
$$

where

$$
e_{n}(\lambda)=\frac{\lambda+\frac{i n}{2}}{\lambda-\frac{i n}{2}}
$$

We restrict the solutions of the BA equations as follows:

$$
\operatorname{Re}\left(\lambda_{\alpha}\right) \geq 0, \quad \lambda_{\alpha} \neq 0, \infty
$$

Within the coordinate Bethe Ansatz approach, these restrictions can be understood by examining the Bethe wavefunction. Alcaraz et $a .^{21}$ write the wavefunction in terms of momenta $\left\{k_{\alpha}\right\}$, which are related to our rapidities $\left\{\lambda_{\alpha}\right\}$ by $\exp \left(i k_{\alpha}\right)=\left(\lambda_{\alpha}+\frac{i}{2}\right) /\left(\lambda_{\alpha}-\frac{i}{2}\right)$. Because of the periodicity $\operatorname{Re}\left(k_{\alpha}\right) \rightarrow \operatorname{Re}\left(k_{\alpha}\right)+2 \pi$ of the wavefunction, one can make the restriction $-\pi \leq \operatorname{Re}\left(k_{\alpha}\right) \leq \pi$. Moreover, changing the sign of (any one) $k_{\alpha}$ results in a change of sign of the wavefunction, and so does not lead to a new independent Bethe state. Thus, we can make the further restriction $0 \leq R e\left(k_{\alpha}\right) \leq \pi$. Finally, one can verify that the wavefunction vanishes identically for $k_{\alpha}=0, \pi$. Translating the restrictions $0 \leq R e\left(k_{\alpha}\right) \leq \pi, \quad k_{\alpha} \neq 0, \pi$ in terms of $\lambda_{\alpha}$ leads to Eq. (3.8) above. See also Refs. 9, 21, $24,26,28$. 
We adopt the "string hypothesis", which states that in the thermodynamic $(N \rightarrow \infty)$ limit, all the solutions are collections of $M_{n}$ strings of length $n$ of the form (for $M_{n}>0$ )

$$
\lambda_{\alpha}^{(n, j)}=\lambda_{\alpha}^{n}+i\left(\frac{n+1}{2}-j\right)
$$

where $j=1, \cdots, n ; \alpha=1, \cdots, M_{n} ; n=1, \cdots, \infty$; and the centers $\lambda_{\alpha}^{n}$ are real and nonnegative. The total number of $\lambda$ variables is $M=\sum_{n=1}^{\infty} n M_{n}$.

Implementing this hypothesis in the BA equations (3.6), and then (following Takahashi ${ }^{29}$ and Gaudin ${ }^{30}$, and using the notation of Tsvelick and Wiegmann ${ }^{31}$ ) forming the product $\prod_{j=1}^{n}$ over the imaginary parts of the strings, we obtain a set of equations for the centers $\lambda_{\alpha}^{n}:$

$$
\begin{gathered}
e_{n}\left(\lambda_{\alpha}^{n}\right)^{2 N+1} \prod_{l=1}^{d\left(n, 2 \xi_{+}-1\right)} e_{n+2 \xi_{+}-2 l}\left(\lambda_{\alpha}^{n}\right) \prod_{l=1}^{d\left(n, 2 \xi_{-}-1\right)} e_{n+2 \xi_{-}-2 l}\left(\lambda_{\alpha}^{n}\right) \\
=(-)^{n} \prod_{m=1}^{\infty} \prod_{\beta=1}^{M_{m}} E_{n m}\left(\lambda_{\alpha}^{n}-\lambda_{\beta}^{m}\right) E_{n m}\left(\lambda_{\alpha}^{n}+\lambda_{\beta}^{m}\right),
\end{gathered}
$$

where

$$
E_{n m}(\lambda)=e_{|n-m|}(\lambda) e_{|n-m|+2}^{2}(\lambda) \cdots e_{n+m-2}^{2}(\lambda) e_{n+m}(\lambda)
$$

and

$$
d(n, x)=\left\{\begin{array}{cl}
\min (n, x) & \text { if } x=\text { integer } \\
n & \text { if } x \neq \text { integer }
\end{array}\right.
$$

In obtaining the above result, we use the lemmas

$$
\begin{gathered}
\prod_{j=1}^{n} e_{x}\left(\lambda_{\alpha}^{(n, j)}\right)=\prod_{l=1}^{d(n, x)} e_{n+x+1-2 l}\left(\lambda_{\alpha}^{n}\right), \\
\prod_{j=1}^{n} \prod_{k=1}^{m} e_{2}\left(\lambda_{\alpha}^{(n, j)}-\lambda_{\beta}^{(m, k)}\right)=E_{n m}\left(\lambda_{\alpha}^{n}-\lambda_{\beta}^{m}\right) .
\end{gathered}
$$

Since the equations (3.10) involve only products of phases, it is useful to take the logarithm:

$$
(2 N+1) q_{n}\left(\lambda_{\alpha}^{n}\right)+\sum_{l=1}^{d\left(n, 2 \xi_{+}-1\right)} q_{n+2 \xi_{+}-2 l}\left(\lambda_{\alpha}^{n}\right)+\sum_{l=1}^{d\left(n, 2 \xi_{-}-1\right)} q_{n+2 \xi_{-}-2 l}\left(\lambda_{\alpha}^{n}\right)
$$




$$
=2 \pi J_{\alpha}^{n}+\sum_{m=1}^{\infty} \sum_{\beta=1}^{M_{m}}\left[\Xi_{n m}\left(\lambda_{\alpha}^{n}-\lambda_{\beta}^{m}\right)+\Xi_{n m}\left(\lambda_{\alpha}^{n}+\lambda_{\beta}^{m}\right)\right],
$$

where $q_{n}(\lambda)$ are odd monotonic-increasing functions defined by

$$
q_{n}(\lambda)=\pi+i \ln e_{n}(\lambda), \quad-\pi<q_{n}(\lambda) \leq \pi
$$

and $\Xi_{n m}(\lambda)$ are given by

$$
\Xi_{n m}(\lambda)=\left(1-\delta_{n m}\right) q_{|n-m|}(\lambda)+2 q_{|n-m|+2}(\lambda)+\cdots+2 q_{n+m-2}(\lambda)+q_{n+m}(\lambda) .
$$

Moreover, $J_{\alpha}^{n}$ are integers in the range

$$
J_{\min }^{n} \leq J_{\alpha}^{n} \leq J_{\max }^{n}
$$

where, for the case $\xi_{ \pm}=\infty$,

$$
J_{m i n}^{n}=1, \quad J_{m a x}^{n}=N+M_{n}-2 \sum_{m=1}^{\infty} \min (m, n) M_{m}
$$

There are similar formulas for the case $\xi_{ \pm} \neq \infty$. (See also Refs. 21,28.) We assume that the integers $\left\{J_{\alpha}^{n}\right\}$ can be regarded as quantum numbers of the model: for every set $\left\{J_{\alpha}^{n}\right\}$ in the range (3.18) (no two of which are identical), there is a unique solution $\left\{\lambda_{\alpha}^{n}\right\}$ (no two of which are identical) of Eq. (3.15).

For definiteness, we henceforth consider the case that $2 \xi_{ \pm}$is not an integer, and therefore, $d\left(n, 2 \xi_{ \pm}-1\right)=n$ in Eq. (3.15). Moreover, for later convenience, we further restrict $\xi_{ \pm}>1 / 2$. (See Eq. (4.10) below.)

Evidently, in order to calculate the boundary $S$ matrices, we must investigate the low-lying excitations of the spin chain. However, as a useful warm-up exercise, we first consider the ground state. 


\section{Ground state}

As discussed in Ref. 21, the ground state is an $S^{z}=0$ state characterized by a filled "Fermi sea" of strings of length 1 . That is, $M_{1}=\frac{N}{2}$ and $M_{n}=0$ for $n>1$. (We consider the case that $N$ is even.) We define the "counting" function $h(\lambda)$ by

$$
h(\lambda)=\frac{1}{2 \pi}\left\{(2 N+1) q_{1}(\lambda)+q_{2 \xi_{+}-1}(\lambda)+q_{2 \xi_{-}-1}(\lambda)-\sum_{\beta=1}^{\frac{N}{2}}\left[q_{2}\left(\lambda-\lambda_{\beta}^{1}\right)+q_{2}\left(\lambda+\lambda_{\beta}^{1}\right)\right]\right\},
$$

so that the BA equations (3.15) for $n=1$ are

$$
h\left(\lambda_{\alpha}^{1}\right)=J_{\alpha}^{1}, \quad \alpha=1, \cdots, \frac{N}{2}
$$

We define the root density $\rho(\lambda)$ by

$$
\rho(\lambda)=\frac{1}{N} \frac{d h(\lambda)}{d \lambda}
$$

In order to control terms of order $1 / N$, one must exercise care when passing from the sum in (4.1) to an integral. In particular, as shown in the Appendix (see Eq. (A.12)), there is an additional term of order $1 / N$. Hence, the root density obeys the integral equation

$$
\begin{aligned}
\rho(\lambda)= & 2 a_{1}(\lambda)-\int_{0}^{\infty} d \lambda^{\prime}\left[a_{2}\left(\lambda-\lambda^{\prime}\right)+a_{2}\left(\lambda+\lambda^{\prime}\right)\right] \rho\left(\lambda^{\prime}\right) \\
& +\frac{1}{N}\left[a_{1}(\lambda)+a_{2}(\lambda)+a_{2 \xi_{+}-1}(\lambda)+a_{2 \xi_{-}-1}(\lambda)\right], \quad \lambda>0
\end{aligned}
$$

(plus terms that are higher order in $1 / N)$, where

$$
a_{n}(\lambda)=\frac{1}{2 \pi} \frac{d q_{n}(\lambda)}{d \lambda}=\frac{1}{2 \pi} \frac{n}{\lambda^{2}+\frac{n^{2}}{4}}=\frac{1}{2 \pi} \int_{-\infty}^{\infty} d \omega e^{-i \omega \lambda} e^{-n|\omega| / 2}, \quad n>0
$$

In order to solve this equation, it is convenient to extend the domain of the root density to negative values of $\lambda$, such that it is an even function of $\lambda$. That is, we introduce the symmetric density $\rho_{s}(\lambda)$

$$
\rho_{s}(\lambda)=\left\{\begin{array}{cc}
\rho(\lambda) & \lambda>0 \\
\rho(-\lambda) & \lambda<0
\end{array}\right.
$$


This density satisfies the linear integral equation

$$
\rho_{s}=2 a_{1}-a_{2} * \rho_{s}+\frac{1}{N}\left(a_{1}+a_{2}+a_{2 \xi_{+}-1}+a_{2 \xi_{-}-1}\right)
$$

where $*$ denotes the convolution

$$
(f * g)(\lambda)=\int_{-\infty}^{\infty} d \lambda^{\prime} f\left(\lambda-\lambda^{\prime}\right) g\left(\lambda^{\prime}\right)
$$

The solution is readily found by Fourier transforms, and is given by

$$
\rho_{s}(\lambda)=2 s(\lambda)+\frac{1}{N}\left[s(\lambda)+J(\lambda)+J_{+}(\lambda)+J_{-}(\lambda)\right]
$$

where

$$
\begin{aligned}
s(\lambda) & =\frac{1}{2 \operatorname{ch} \pi \lambda}=\frac{1}{2 \pi} \int_{-\infty}^{\infty} d \omega e^{-i \omega \lambda} \frac{e^{-|\omega| / 2}}{1+e^{-|\omega|}} \\
J(\lambda) & =\left(a_{1} * s\right)(\lambda)=\frac{1}{2 \pi} \int_{-\infty}^{\infty} d \omega e^{-i \omega \lambda} \frac{e^{-|\omega|}}{1+e^{-|\omega|}} \\
J_{ \pm}(\lambda) & =\left(a_{2 \xi_{ \pm}-2} * s\right)(\lambda)=\frac{1}{2 \pi} \int_{-\infty}^{\infty} d \omega e^{-i \omega \lambda} \frac{e^{-\left(\xi_{ \pm}-\frac{1}{2}\right)|\omega|}}{1+e^{-|\omega|}}
\end{aligned}
$$

We calculate the ground state energy density $e_{N}^{v a c}=E / N$ with the help of Eq. (3.4). Again exercising care in passing from a sum to an integral, we obtain (to order $1 / N$ )

$$
\begin{aligned}
e_{N}^{v a c} & =-\frac{\pi}{N} \sum_{\alpha=1}^{\frac{N}{2}} a_{1}\left(\lambda_{\alpha}^{1}\right) \\
& =-\frac{\pi}{2}\left[\int_{-\infty}^{\infty} d \lambda a_{1}(\lambda) \rho_{s}(\lambda)-\frac{1}{N} a_{1}(0)\right] \\
& =e_{\infty}^{v a c}+\frac{f}{N}
\end{aligned}
$$

where $e_{\infty}^{v a c}$ is the energy density of the infinite chain, and the surface energy $f$ of the chain is given by

$$
f=\frac{\pi}{2}\left\{s(0)-\int_{-\infty}^{\infty} d \lambda a_{1}(\lambda)\left[s(\lambda)+J_{+}(\lambda)+J_{-}(\lambda)\right]\right\}
$$


Our results for the root density (4.9) and the surface energy (4.12) agree with those of Ref. 26.

\section{Excited states and $S$ matrix}

As shown by Faddeev and Takhtajan ${ }^{25}$, the isotropic antiferromagnetic Heisenberg chain with periodic boundary conditions has spin $1 / 2$ excitations ("kinks" or "spinons"). Following their approach, we consider the case that $N$ is even, and therefore the number of such spinons is even. Hence, the lowest-lying excited states have two spinons, and there are four such states: the triplet $(S=1)$ and singlet $(S=0)$ states.

For the open spin chain, the bulk terms in the Hamiltonian are $S U(2)$-invariant; hence, the excitations are still spin 1/2 spinons, and there are four degenerate states with two spinons. However, the boundary terms break the $S U(2)$ symmetry, and so the total spin is no longer a good quantum number. We now proceed to investigate the excited states, which we classify by their $S^{z}$ eigenvalue. (Excited states of the open spin $1 / 2$ chain have also been studied in Refs. 9, 28.)

$S^{z}=1$ state

The $S^{z}=1$ state is characterized by two holes in the sea of strings of length 1 , and no strings of greater length. That is, $M_{1}=\frac{N}{2}-1$ and $M_{n}=0$ for $n>1$. The counting function $h^{(1)}(\lambda)$ is given by

$h^{(1)}(\lambda)=\frac{1}{2 \pi}\left\{(2 N+1) q_{1}(\lambda)+q_{2 \xi_{+}-1}(\lambda)+q_{2 \xi_{-}-1}(\lambda)-\sum_{\beta=1}^{\frac{N}{2}-1}\left[q_{2}\left(\lambda-\lambda_{\beta}^{1}\right)+q_{2}\left(\lambda+\lambda_{\beta}^{1}\right)\right]\right\}$.

The hole rapidities $\tilde{\lambda}_{1}, \tilde{\lambda}_{2}$ are given by

$$
h^{(1)}\left(\tilde{\lambda}_{\alpha}\right)=\tilde{J}_{\alpha}, \quad \alpha=1,2,
$$


where $\tilde{J}_{1}, \tilde{J}_{2}$ are integers in the range

$$
J_{\min }^{1} \leq \tilde{J}_{1}, \tilde{J}_{2} \leq J_{\max }^{1}
$$

and which are not in the set of integers $\left\{J_{\alpha}^{1}\right\}$ corresponding to $\left\{\lambda_{\alpha}^{1}\right\}$.

We define the quantity $\sigma^{(1)}(\lambda)$

$$
\sigma^{(1)}(\lambda)=\frac{1}{N} \frac{d h^{(1)}(\lambda)}{d \lambda}
$$

which is the density of roots plus the density of holes. * We proceed as in the case of the ground state, being careful when passing from the sum to an integral (see Eq. (A.12)), and we obtain the integral equation

$$
\begin{aligned}
\sigma^{(1)}(\lambda) & =2 a_{1}(\lambda)-\int_{0}^{\infty} d \lambda^{\prime}\left[a_{2}\left(\lambda-\lambda^{\prime}\right)+a_{2}\left(\lambda+\lambda^{\prime}\right)\right] \sigma^{(1)}\left(\lambda^{\prime}\right) \\
& +\frac{1}{N}\left\{a_{1}(\lambda)+a_{2}(\lambda)+a_{2 \xi_{+}-1}(\lambda)+a_{2 \xi_{-}-1}(\lambda)\right. \\
& \left.+\sum_{\alpha=1}^{2}\left[a_{2}\left(\lambda-\tilde{\lambda}_{\alpha}\right)+a_{2}\left(\lambda+\tilde{\lambda}_{\alpha}\right)\right]\right\}, \quad \lambda>0
\end{aligned}
$$

(plus terms that are higher order in $1 / N)$. The symmetric density $\sigma_{s}^{(1)}(\lambda)$ defined by

$$
\sigma_{s}^{(1)}(\lambda)=\left\{\begin{array}{cc}
\sigma^{(1)}(\lambda) & \lambda>0 \\
\sigma^{(1)}(-\lambda) & \lambda<0
\end{array},\right.
$$

is therefore given by

$$
\sigma_{s}^{(1)}(\lambda)=2 s(\lambda)+\frac{1}{N} r^{(1)}(\lambda)
$$

where $r^{(1)}(\lambda)$ is given by

$$
r^{(1)}(\lambda)=s(\lambda)+J(\lambda)+J_{+}(\lambda)+J_{-}(\lambda)+\sum_{\alpha=1}^{2}\left[J\left(\lambda-\tilde{\lambda}_{\alpha}\right)+J\left(\lambda+\tilde{\lambda}_{\alpha}\right)\right]
$$

* Since $\sigma^{(1)}$ depends also on the hole rapidities, the notation $\sigma^{(1)}\left(\lambda, \tilde{\lambda}_{1}, \tilde{\lambda}_{2}\right)$ would be more accurate. However, following the usual practice, we suppress the dependence on the hole rapidities. 
(plus terms that are higher order in $1 / N)$. The functions $s(\lambda), J(\lambda)$, and $J_{ \pm}(\lambda)$ are defined in Eq. (4.10).

The energy density $e_{N}=E / N$ is given by

$$
e_{N}=e_{N}^{v a c}+\frac{1}{N} \sum_{\alpha=1}^{2} \varepsilon\left(\tilde{\lambda}_{\alpha}\right)
$$

where $e_{N}^{v a c}$ is the ground state energy density (4.11), and the spinon energy $\varepsilon(\lambda)$ is given by

$$
\varepsilon(\lambda)=\pi s(\lambda)
$$

(plus terms that are higher order in $1 / N)$.

The spinons of the antiferromagnetic Heisenberg chain with periodic boundary conditions have the same energy, and have momentum $p(\lambda)$ given by ${ }^{25}$

$$
p(\lambda)=\tan ^{-1} \operatorname{sh}(\pi \lambda)-\frac{\pi}{2} .
$$

For the open spin chain, we define $p(\lambda)$ by this equation. Note that

$$
\frac{d p}{d \lambda}=2 \pi s(\lambda)
$$

We come now to the calculation of the $S$ matrix. As already noted in the Introduction, our approach is a generalization of the Korepin-Andrei-Destri method ${ }^{22,23}$. From Eqs. (5.4), (5.7), and (5.12), we obtain the relation

$$
\frac{1}{N} \frac{d h^{(1)}}{d \lambda}=\frac{1}{\pi} \frac{d p}{d \lambda}+\frac{1}{N} r^{(1)}(\lambda)
$$

Integrating with respect to $\lambda$, we obtain

$$
\frac{2 \pi}{N} h^{(1)}(\lambda)=2 p(\lambda)+\frac{\pi}{N} \int_{-\infty}^{\infty} d \lambda^{\prime} \epsilon\left(\lambda-\lambda^{\prime}\right) r^{(1)}\left(\lambda^{\prime}\right)+\pi,
$$

where $\epsilon(x)=\operatorname{sign} x=x /|x|$. Finally, we evaluate this expression at the spinon rapidity $\tilde{\lambda}_{1}$, recalling from Eq. (5.2) that $h\left(\tilde{\lambda}_{1}\right)=\tilde{J}_{1}$. Thus,

$$
\frac{2 \pi}{N} \tilde{J}_{1}=2 p\left(\tilde{\lambda}_{1}\right)+\frac{\pi}{N} \int_{-\infty}^{\infty} d \lambda \epsilon\left(\tilde{\lambda}_{1}-\lambda\right) r^{(1)}(\lambda)+\pi .
$$


On the other hand, let us recall the quantization condition (2.11) for two particles with factorized scattering on an interval of length $L$. For the spin chain, the number of spins $N$ replaces $L$, and the "particles" are in fact spin $1 / 2$ spinons of rapidities $\tilde{\lambda}_{1}$ and $\tilde{\lambda}_{2}$. Thus, we have

$$
e^{i 2 p\left(\tilde{\lambda}_{1}\right) N} R_{12}\left(\tilde{\lambda}_{1}-\tilde{\lambda}_{2}\right) K_{1}\left(\tilde{\lambda}_{1}, \xi_{-}\right) R_{21}\left(\tilde{\lambda}_{1}+\tilde{\lambda}_{2}\right) K_{1}\left(\tilde{\lambda}_{1}, \xi_{+}\right)=1 .
$$

The bulk 2-particle $S$ matrix is $S U(2)$-invariant, and is given by ${ }^{25}$

$$
R(\lambda)=\frac{\Gamma\left(\frac{-i \lambda}{2}+\frac{1}{2}\right)}{\Gamma\left(\frac{i \lambda}{2}+\frac{1}{2}\right)} \frac{\Gamma\left(\frac{i \lambda}{2}+1\right)}{\Gamma\left(\frac{-i \lambda}{2}+1\right)} \frac{(\lambda \mathcal{I}-i \mathcal{P})}{(\lambda-i)}
$$

where $\mathcal{I}$ and $\mathcal{P}$ are the $4 \times 4$ identity and permutation matrices, respectively. This matrix has the following form

$$
R(\lambda)=\left(\begin{array}{cccc}
a(\lambda) & 0 & 0 & 0 \\
0 & b(\lambda) & c(\lambda) & 0 \\
0 & c(\lambda) & b(\lambda) & 0 \\
0 & 0 & 0 & a(\lambda)
\end{array}\right)
$$

with $a(\lambda)=b(\lambda)+c(\lambda)$.

The $U(1)$ symmetry of the Hamiltonian's boundary terms implies that the boundary $S$ matrix is of the form

$$
K(\lambda, \xi)=\left(\begin{array}{cc}
\alpha(\lambda, \xi) & 0 \\
0 & \beta(\lambda, \xi)
\end{array}\right)
$$

Our task is to explicitly determine the matrix elements $\alpha(\lambda, \xi)$ and $\beta(\lambda, \xi)$.

For the $S^{z}=1$ state, the quantization condition (5.16) implies

$$
e^{i 2 p\left(\tilde{\lambda}_{1}\right) N} a\left(\tilde{\lambda}_{1}-\tilde{\lambda}_{2}\right) \alpha\left(\tilde{\lambda}_{1}, \xi_{-}\right) a\left(\tilde{\lambda}_{1}+\tilde{\lambda}_{2}\right) \alpha\left(\tilde{\lambda}_{1}, \xi_{+}\right)=1 .
$$

In terms of the bulk $\left(\phi^{(1)}\right)$ and boundary $(\varphi)$ phase shifts defined by

$$
a(\lambda)=e^{i \phi^{(1)}(\lambda)}, \quad \alpha(\lambda, \xi)=e^{i \varphi(\lambda, \xi)},
$$


respectively, the quantization condition (5.20) becomes

$$
\frac{2 \pi}{N} m=2 p\left(\tilde{\lambda}_{1}\right)+\frac{1}{N} \Phi^{(1)}
$$

where $m$ is an integer, and the total phase shift $\Phi^{(1)}$ is given by

$$
\Phi^{(1)}=\phi^{(1)}\left(\tilde{\lambda}_{1}-\tilde{\lambda}_{2}\right)+\phi^{(1)}\left(\tilde{\lambda}_{1}+\tilde{\lambda}_{2}\right)+\varphi\left(\tilde{\lambda}_{1}, \xi_{-}\right)+\varphi\left(\tilde{\lambda}_{1}, \xi_{+}\right) .
$$

Comparing our Bethe Ansatz result (5.15) with the quantization condition (5.22), we see that the total phase shift $\Phi^{(1)}$ is given by

$$
\Phi^{(1)}=\pi \int_{-\infty}^{\infty} d \lambda \epsilon\left(\tilde{\lambda}_{1}-\lambda\right) r^{(1)}(\lambda)
$$

It is convenient to evaluate the derivative of this expression,

$$
\begin{aligned}
\frac{d \Phi^{(1)}}{d \tilde{\lambda}_{1}}= & 2 \pi\left[s\left(\tilde{\lambda}_{1}\right)+J\left(\tilde{\lambda}_{1}\right)+2 J\left(2 \tilde{\lambda}_{1}\right)+J_{+}\left(\tilde{\lambda}_{1}\right)+J_{-}\left(\tilde{\lambda}_{1}\right)\right. \\
& \left.+J\left(\tilde{\lambda}_{1}-\tilde{\lambda}_{2}\right)+J\left(\tilde{\lambda}_{1}+\tilde{\lambda}_{2}\right)\right]
\end{aligned}
$$

In obtaining this result, we have used Eq. (5.8) for $r^{(1)}(\lambda)$; and we have remembered to differentiate also $r^{(1)}(\lambda)$ in Eq. (5.24). The bulk phase shift $\phi^{(1)}(\lambda)$ given by Eqs. (5.17),(5.18),(5.21) satisfies

$$
\frac{d \phi^{(1)}(\lambda)}{d \lambda}=2 \pi J(\lambda)
$$

In view also of Eq. (5.23), we see that the boundary phase shift $\varphi\left(\lambda, \xi_{ \pm}\right)$satisfies

$$
\frac{d \varphi\left(\lambda, \xi_{ \pm}\right)}{d \lambda}=\pi\left[s(\lambda)+J(\lambda)+2 J(2 \lambda)+2 J_{ \pm}(\lambda)\right]
$$

The phase shift is now readily evaluated by expressing the RHS in terms of the Fourier transform, and using the identity

$$
\int_{0}^{\infty} \frac{d \omega}{\omega} \frac{e^{-\nu \omega}}{1+e^{-\omega}}=\ln \left(\frac{\Gamma\left(\frac{\nu}{2}\right)}{\Gamma\left(\frac{\nu+1}{2}\right)}\right), \quad \operatorname{Re} \nu>0,
$$


and also the duplication formula for the gamma function,

$$
2^{2 z-1} \Gamma(z) \Gamma\left(z+\frac{1}{2}\right)=\pi^{\frac{1}{2}} \Gamma(2 z)
$$

The result for the boundary $S$ matrix element (up to a multiplicative constant) is

$$
\begin{aligned}
& \alpha\left(\lambda, \xi_{ \pm}\right)=e^{i \varphi\left(\lambda, \xi_{ \pm}\right)}=\frac{\Gamma\left(\frac{-i \lambda}{2}+\frac{1}{4}\right)}{\Gamma\left(\frac{i \lambda}{2}+\frac{1}{4}\right)} \frac{\Gamma\left(\frac{i \lambda}{2}+1\right)}{\Gamma\left(\frac{-i \lambda}{2}+1\right)} \frac{\Gamma\left(\frac{-i \lambda}{2}+\frac{1}{4}\left(2 \xi_{ \pm}-1\right)\right)}{\Gamma\left(\frac{i \lambda}{2}+\frac{1}{4}\left(2 \xi_{ \pm}-1\right)\right)} \frac{\Gamma\left(\frac{i \lambda}{2}+\frac{1}{4}\left(2 \xi_{ \pm}+1\right)\right)}{\Gamma\left(\frac{-i \lambda}{2}+\frac{1}{4}\left(2 \xi_{ \pm}+1\right)\right)} . \\
& S^{z}=-1 \text { state }
\end{aligned}
$$

To determine the remaining element $\beta(\lambda, \xi)$ of the boundary $S$ matrix, we consider the $S^{z}=-1$ state. This state is most easily described by changing the pseudovacuum. Hence, instead of working with the states (3.1), we work now with

$$
\mathcal{C}\left(\lambda_{1}\right) \mathcal{C}\left(\lambda_{2}\right) \cdots \mathcal{C}\left(\lambda_{M}\right) \omega^{-}
$$

where $\omega^{-}$is the ferromagnetic vacuum state with all spins down,

$$
\mathcal{B}(\lambda) \omega^{-}=0
$$

Sklyanin has shown ${ }^{4}$ that $\left\{\lambda_{\alpha}\right\}$ satisfy the same BA equations (3.3) as before, except for the replacement of $\xi_{ \pm}$by $-\xi_{ \pm}$. The energy eigenvalues are given by the same expression (3.4), and the $S^{z}$ eigenvalues are now given by

$$
S^{z}=M-\frac{N}{2}
$$

The $S^{z}=-1$ state now consists of two holes in the sea of strings of length 1 , and no strings of greater length; i.e., $M_{1}=\frac{N}{2}-1$ and $M_{n}=0$ for $n>1$. The calculation of the density of roots plus holes is exactly the same as for the $S^{z}=1$ state, except that we must 
track the change $\xi_{ \pm} \rightarrow-\xi_{ \pm}$. We find that the density is given by Eqs. (5.7) and (5.8), except that $J_{ \pm}(\lambda)$ is now given by

$$
J_{ \pm}(\lambda)=-\left(a_{2 \xi_{ \pm}} * s\right)(\lambda)=-\frac{1}{2 \pi} \int_{-\infty}^{\infty} d \omega e^{-i \omega \lambda} \frac{e^{-\left(\xi_{ \pm}+\frac{1}{2}\right)|\omega|}}{1+e^{-|\omega|}}
$$

Moreover, the quantization condition (5.16) and the form (5.18),(5.19) of the $S$ matrices imply

$$
e^{i 2 p\left(\tilde{\lambda}_{1}\right) N} a\left(\tilde{\lambda}_{1}-\tilde{\lambda}_{2}\right) \beta\left(\tilde{\lambda}_{1}, \xi_{-}\right) a\left(\tilde{\lambda}_{1}+\tilde{\lambda}_{2}\right) \beta\left(\tilde{\lambda}_{1}, \xi_{+}\right)=1
$$

Introducing the phase shift $\psi(\lambda, \xi)$ by $\beta(\lambda, \xi)=\exp (i \psi(\lambda, \xi))$, we find that $\psi\left(\lambda, \xi_{ \pm}\right)$ obeys Eq. (5.27), with $J_{ \pm}$given by Eq. (5.34). We conclude that the element $\beta(\lambda, \xi)$ of the boundary $S$ matrix is given (up to a multiplicative constant) by

$$
\beta\left(\lambda, \xi_{ \pm}\right)=-\frac{\lambda+i\left(\xi_{ \pm}-\frac{1}{2}\right)}{\lambda-i\left(\xi_{ \pm}-\frac{1}{2}\right)} \alpha(\lambda)
$$

where $\alpha(\lambda)$ is given by Eq. (5.30). This completes the derivation of the result (1.7), (1.8) for the boundary $S$ matrix.

$S^{z}=0$ states

We have already succeeded to determine the boundary $S$ matrix. Nevertheless, a good check on this result and on the general formalism is provided by analyzing the $S^{z}=0$ states. In particular, with the help of expressions for the densities, we can determine the "momentum" $p\left(\tilde{\lambda}_{1}\right)$ of a spinon in terms of its deviation from the free-particle value. Substituting this result, as well as the known results for $R(\lambda)$ and $K\left(\lambda, \xi_{ \pm}\right)$, into the quantization condition (5.16), we obtain a consistency condition.

To this end, we consider the $S^{z}=0$ state consisting of two holes in the sea of strings of length 1 , and also one string of length 2 ; i.e., $M_{1}=\frac{N}{2}-2, M_{2}=1$, and $M_{n}=0$ for $n>2$. For $\xi_{ \pm} \rightarrow \infty$, this is the spin-singlet $\left(S=S^{z}=0\right)$ state. The counting function 
$h^{(0)}(\lambda)$ is given by (see the BA equation (3.15) with $n=1$ )

$$
\begin{aligned}
h^{(0)}(\lambda) & =\frac{1}{2 \pi}\left\{(2 N+1) q_{1}(\lambda)+q_{2 \xi_{+}-1}(\lambda)+q_{2 \xi_{-}-1}(\lambda)-\sum_{\beta=1}^{\frac{N}{2}-2}\left[q_{2}\left(\lambda-\lambda_{\beta}^{1}\right)+q_{2}\left(\lambda+\lambda_{\beta}^{1}\right)\right]\right. \\
& \left.-q_{1}\left(\lambda-\lambda_{0}\right)-q_{3}\left(\lambda-\lambda_{0}\right)-q_{1}\left(\lambda+\lambda_{0}\right)-q_{3}\left(\lambda+\lambda_{0}\right)\right\}
\end{aligned}
$$

where $\lambda_{0} \equiv \lambda_{1}^{2}$ is the position of the center of the string of length 2 . The density $\sigma^{(0)}(\lambda)$ (defined analogously as in Eq. (5.4)) obeys the integral equation

$$
\begin{aligned}
\sigma^{(0)}(\lambda) & =2 a_{1}(\lambda)-\int_{0}^{\infty} d \lambda^{\prime}\left[a_{2}\left(\lambda-\lambda^{\prime}\right)+a_{2}\left(\lambda+\lambda^{\prime}\right)\right] \sigma^{(0)}\left(\lambda^{\prime}\right) \\
& +\frac{1}{N}\left\{a_{1}(\lambda)+a_{2}(\lambda)+a_{2 \xi_{+}-1}(\lambda)+a_{2 \xi_{-}-1}(\lambda)\right. \\
& +\sum_{\alpha=1}^{2}\left[a_{2}\left(\lambda-\tilde{\lambda}_{\alpha}\right)+a_{2}\left(\lambda+\tilde{\lambda}_{\alpha}\right)\right] \\
& \left.-a_{1}\left(\lambda-\lambda_{0}\right)-a_{3}\left(\lambda-\lambda_{0}\right)-a_{1}\left(\lambda+\lambda_{0}\right)-a_{3}\left(\lambda+\lambda_{0}\right)\right\}, \quad \lambda>0
\end{aligned}
$$

(plus terms that are higher order in $1 / N)$. We conclude that the symmetric density $\sigma_{s}^{(0)}(\lambda)$ (defined analogously as in Eq. (5.6)) is given by

$$
\sigma_{s}^{(0)}(\lambda)=2 s(\lambda)+\frac{1}{N} r^{(0)}(\lambda)
$$

where

$$
r^{(0)}(\lambda)=r^{(1)}(\lambda)-\left[a_{1}\left(\lambda-\lambda_{0}\right)+a_{1}\left(\lambda+\lambda_{0}\right)\right]
$$

and $r^{(1)}(\lambda)$ is given in Eq. (5.8).

The position $\lambda_{0}$ of the center of the string of length 2 can be determined in terms of the positions $\tilde{\lambda}_{1}$ and $\tilde{\lambda}_{2}$ of the holes. We accomplish this using the BA equations (3.15) with $n=2$ :

$$
\begin{aligned}
& (2 N+1) q_{2}\left(\lambda_{0}\right)+q_{2 \xi_{+}}\left(\lambda_{0}\right)+q_{2 \xi_{+}-2}\left(\lambda_{0}\right)+q_{2 \xi_{-}}\left(\lambda_{0}\right)+q_{2 \xi_{-}-2}\left(\lambda_{0}\right) \\
& \quad=2 \pi J_{1}^{2}+\sum_{\beta=1}^{\frac{N}{2}-2}\left[\Xi_{21}\left(\lambda_{0}-\lambda_{\beta}^{1}\right)+\Xi_{21}\left(\lambda_{0}+\lambda_{\beta}^{1}\right)\right]+2 q_{1}\left(\lambda_{0}\right)+q_{2}\left(\lambda_{0}\right) .
\end{aligned}
$$


(We remind the reader that, in terms of the notation in Eq. (3.15), $\lambda_{0}$ is in fact $\lambda_{1}^{2}$.) Passing again from the sum to an integral, and making use of the result (5.39), we are led to the constraint

$$
e_{2 \xi_{+}-2}\left(\lambda_{0}\right) e_{2 \xi_{-}-2}\left(\lambda_{0}\right) e_{1}\left(\lambda_{0}-\tilde{\lambda}_{1}\right) e_{1}\left(\lambda_{0}-\tilde{\lambda}_{2}\right) e_{1}\left(\lambda_{0}+\tilde{\lambda}_{1}\right) e_{1}\left(\lambda_{0}+\tilde{\lambda}_{2}\right)=1
$$

This is considerably more complicated than the corresponding constraint for the periodic chain, which implies $\lambda_{0}^{(\text {periodic })}=\left(\tilde{\lambda}_{1}+\tilde{\lambda}_{2}\right) / 2$.

For the case $\xi_{ \pm}=\infty$, the constraint becomes

$$
e_{1}\left(\lambda_{0}-\tilde{\lambda}_{1}\right) e_{1}\left(\lambda_{0}-\tilde{\lambda}_{2}\right) e_{1}\left(\lambda_{0}+\tilde{\lambda}_{1}\right) e_{1}\left(\lambda_{0}+\tilde{\lambda}_{2}\right)=1
$$

In addition to the solution $\lambda_{0}=0^{*}$, this constraint has the solution

$$
\lambda_{0}=\sqrt{\frac{1}{4}+\frac{1}{2}\left[\left(\tilde{\lambda}_{1}\right)^{2}+\left(\tilde{\lambda}_{2}\right)^{2}\right]} .
$$

For $\xi_{ \pm} \neq \infty$, Eq. (5.42) for $\lambda_{0}$ is not difficult to solve. Nevertheless, the solution is given by a rather cumbersome expression, which we shall not present here.

One can verify that this $S^{z}=0$ state has the same energy $(5.9),(5.10)$ as the $S^{z}=1$ state. We now express the spinon "momentum" $p\left(\tilde{\lambda}_{1}\right)$ in terms of its deviation from the free-particle value. In analogy with the $S^{z}=1$ case, we have

$$
\frac{2 \pi}{N} \tilde{J}_{1}=2 p\left(\tilde{\lambda}_{1}\right)+\frac{\pi}{N} \int_{-\infty}^{\infty} d \lambda \epsilon\left(\tilde{\lambda}_{1}-\lambda\right) r^{(0)}(\lambda)+\pi
$$

Substituting for $r^{(0)}(\lambda)$ the expression (5.40), we obtain the result

$$
2 p\left(\tilde{\lambda}_{1}\right)=\frac{2 \pi}{N} \tilde{J}_{1}+\frac{1}{N}\left[-\Phi^{(1)}+q_{1}\left(\tilde{\lambda}_{1}-\lambda_{0}\right)+q_{1}\left(\tilde{\lambda}_{1}+\lambda_{0}\right)\right]
$$

where $\Phi^{(1)}$ is given by Eq. (5.24).

* The restriction $(3.8)$ can presumably be strengthened to $\operatorname{Re}\left(\lambda_{\alpha}\right)>0$, and thus, we discard this solution. 
We turn now to the quantization condition. For the $S^{z}=0$ states, the quantization condition (5.16) leads to a $2 \times 2$ matrix equation. The two eigenvalues of this matrix are pure phases. (Since the matrix elements of $R(\lambda)$ and $K\left(\lambda, \xi_{ \pm}\right)$are known, these eigenvalues can be computed explicitly. However, for $\xi_{ \pm} \neq \infty$, the actual expressions for the eigenvalues are cumbersome, and we do not give them here.) We consider the eigenvalue $\exp i \Phi^{(0)}$ which for $\xi_{ \pm} \rightarrow \infty$ corresponds to the spin-singlet $\left(S=S^{z}=0\right)$ state. $\nmid$ Hence, the quantization condition implies

$$
e^{i 2 p\left(\tilde{\lambda}_{1}\right) N} e^{i \Phi^{(0)}}=1 .
$$

Substituting the result for $2 p\left(\tilde{\lambda}_{1}\right)$ given in Eq. (5.46) into Eq. (5.47), we obtain the consistency condition

$$
e^{i\left(\Phi^{(0)}-\Phi^{(1)}\right)}=e_{1}\left(\tilde{\lambda}_{1}-\lambda_{0}\right) e_{1}\left(\tilde{\lambda}_{1}+\lambda_{0}\right)
$$

where $\tilde{\lambda}_{1}$ and $\tilde{\lambda}_{2}$ are arbitrary, and $\lambda_{0}$ is a solution of Eq. (5.42).

This relation is readily verified for $\xi_{ \pm}=\infty$. Indeed, for this case, the boundary $S$ matrix is proportional to the identity matrix $(\alpha(\lambda, \infty)=\beta(\lambda, \infty))$. The $2 \times 2$ matrix equation which follows from the quantization condition is readily diagonalized, and the phase shift $\Phi^{(0)}$ is found to be

$$
\Phi^{(0)}=\phi^{(0)}\left(\tilde{\lambda}_{1}-\tilde{\lambda}_{2}\right)+\phi^{(0)}\left(\tilde{\lambda}_{1}+\tilde{\lambda}_{2}\right)+2 \varphi\left(\tilde{\lambda}_{1}, \infty\right) .
$$

Here $\phi^{(0)}(\lambda)$ is the singlet phase shift

$$
b(\lambda)-c(\lambda)=e^{i \phi^{(0)}(\lambda)}=-\left(\frac{\lambda+i}{\lambda-i}\right) e^{i \phi^{(1)}(\lambda)},
$$

where $\phi^{(1)}(\lambda)$ is the triplet phase shift. (See Eqs. (5.17),(5.18),(5.21).) Since

$$
\Phi^{(1)}=\phi^{(1)}\left(\tilde{\lambda}_{1}-\tilde{\lambda}_{2}\right)+\phi^{(1)}\left(\tilde{\lambda}_{1}+\tilde{\lambda}_{2}\right)+2 \varphi\left(\tilde{\lambda}_{1}, \infty\right),
$$

$\dagger$ We implicitly assume that $\Phi^{(0)}$ is a continuous function of $\xi_{ \pm}$. 
it follows that

$$
e^{i\left(\Phi^{(0)}-\Phi^{(1)}\right)}=e_{1}\left(\frac{1}{2}\left(\tilde{\lambda}_{1}-\tilde{\lambda}_{2}\right)\right) e_{1}\left(\frac{1}{2}\left(\tilde{\lambda}_{1}+\tilde{\lambda}_{2}\right)\right) .
$$

The relation (5.48) now follows from the algebraic identity

$$
e_{1}\left(\tilde{\lambda}_{1}-\lambda_{0}\right) e_{1}\left(\tilde{\lambda}_{1}+\lambda_{0}\right)=e_{1}\left(\frac{1}{2}\left(\tilde{\lambda}_{1}-\tilde{\lambda}_{2}\right)\right) e_{1}\left(\frac{1}{2}\left(\tilde{\lambda}_{1}+\tilde{\lambda}_{2}\right)\right)
$$

This identity is true for arbitrary values of $\tilde{\lambda}_{1}$ and $\tilde{\lambda}_{2}$, where $\lambda_{0}$ is given by (5.44).

We have explicitly verified the formula (5.48) also for the case $\xi_{-}=\infty, \xi_{+} \neq \infty$, and presumably it is true in general. This equality provides a nontrivial consistency check of the bulk and boundary $S$ matrices and of the general formalism.

\section{Discussion}

We have demonstrated how the boundary $S$ matrix for the open Heisenberg chain with boundary magnetic fields can be calculated directly from the Bethe Ansatz equations. As yet, this is the only quantum-mechanical calculation of the boundary $S$ matrix. We have restricted our attention in this paper to the case that the bulk terms are $S U(2)$ invariant and the boundary terms are $U(1)$ invariant only for the sake of simplicity. We see no difficulty in extending our calculations to the case that both the bulk terms and the boundary terms are only $U(1)$ invariant. However, the case where the boundary terms have no continuous symmetry cannot be undertaken by this approach until the corresponding Bethe Ansatz solution is found ${ }^{11}$.

The analysis of the $S^{z}=0$ states for the open chain differs significantly from that of the periodic chain. Indeed, for the open chain, we have seen that the position of the center of the string of length 2 is a complicated function of the hole positions $\tilde{\lambda}_{1}$ and $\tilde{\lambda}_{2}$ (as well as the boundary parameters $\xi_{ \pm}$); while for the periodic chain, the center of the string is located midway between the two holes. Naively, one might worry that this leads 
to a breakdown of factorization. However, we have seen that factorization is maintained by virtue of certain nontrivial identities. We expect that a similar situation holds for the periodic chain with four or more holes.

This work was supported in part by the National Science Foundation under Grants PHY-92 22318 and PHY-92 09978.

\section{A. Appendix}

We derive in this Appendix a formula for approximating a sum of the form

$$
\frac{1}{N} \sum_{\alpha=1}^{M_{1}} g\left(\lambda_{\alpha}^{1}\right)
$$

by an integral, to order $1 / N$, for large $N$. Here $g(\lambda)$ is an arbitrary function of $\lambda$ which goes to 0 for $\lambda \rightarrow \infty$. Furthermore, $\left\{\lambda_{\alpha}^{1}\right\}$ are solutions of the BA equations (3.15) with $n=1$. That is,

$$
h\left(\lambda_{\alpha}^{1}\right)=J_{\alpha}^{1}, \quad \alpha=1, \cdots, M_{1},
$$

where $h(\lambda)$ is the appropriate counting function, and $M_{1} \sim N / 2$. There are two key points in our discussion: (1) we use the Euler-Maclaurin formula for approximating sums by integrals; and (2) we use the fact that the solution $\lambda=0$ of the BA equations is excluded. The Euler-Maclaurin formula has been widely used to calculate finite-size corrections in Bethe Ansatz systems. (See, e.g., Refs. 26, 32.)

Let us consider the case that there are $\nu$ holes, with rapidities $\left\{\tilde{\lambda}_{\alpha}\right\}$

$$
h\left(\tilde{\lambda}_{\alpha}\right)=\tilde{J}_{\alpha}, \quad \alpha=1, \cdots, \nu .
$$

We denote the union of the sets $\left\{\lambda_{\alpha}^{1}\right\}$ and $\left\{\tilde{\lambda}_{\alpha}\right\}$ by $\left\{\Lambda_{\alpha}\right\}$, with $\alpha=1, \cdots, I$, where $I=M_{1}+\nu$. Similarly, we denote the union of the sets $\left\{J_{\alpha}^{1}\right\}$ and $\left\{\tilde{J}_{\alpha}\right\}$ by $\left\{j_{\alpha}\right\}$. Evidently,

$$
h\left(\Lambda_{\alpha}\right)=j_{\alpha}=\alpha, \quad \alpha=1, \cdots, I .
$$


As noted in Eq. (3.8), $\Lambda_{\alpha}>0$. Nevertheless, it is useful to introduce $\Lambda_{0} \equiv 0$. Since $h(0)=0$, the corresponding integer is $j_{0}=0$.

Instead of evaluating the sum (A.1) directly, it is convenient to first evaluate the sum

$$
\frac{1}{N} \sum_{\alpha=0}^{I} g\left(\Lambda_{\alpha}\right) .
$$

We approximate this sum using the Euler-Maclaurin formula (see, e.g., Ref. 33)

$$
\sum_{k=0}^{n} f(a+k \delta)=\frac{1}{\delta} \int_{a}^{a+n \delta} d x f(x)+\frac{1}{2}[f(a)+f(a+n \delta)]+\cdots
$$

Indeed, transforming to a sum over equidistant points by means of the change of variables $h(\lambda)=j$, we obtain

$$
\begin{aligned}
\frac{1}{N} \sum_{\alpha=0}^{I} g\left(\Lambda_{\alpha}\right) & =\frac{1}{N} \sum_{\alpha=0}^{I} g\left(h^{-1}\left(j_{\alpha}\right)\right) \\
& =\frac{1}{N} \int_{j_{0}}^{j_{I}} d j g\left(h^{-1}(j)\right)+\frac{1}{2 N}\left[g\left(h^{-1}\left(j_{0}\right)\right)+g\left(h^{-1}\left(j_{I}\right)\right)\right]
\end{aligned}
$$

(plus terms that are higher order in $1 / N)$. Introducing the density $\sigma(\lambda)$

$$
\sigma(\lambda)=\frac{1}{N} \frac{d h(\lambda)}{d \lambda}
$$

we obtain

$$
\frac{1}{N} \sum_{\alpha=0}^{I} g\left(\Lambda_{\alpha}\right)=\int_{0}^{\Lambda_{I}} d \lambda \sigma(\lambda) g(\lambda)+\frac{1}{2 N}\left[g(0)+g\left(\Lambda_{I}\right)\right] .
$$

By definition of $\left\{\Lambda_{\alpha}\right\}$,

$$
\sum_{\alpha=0}^{I} g\left(\Lambda_{\alpha}\right)=g(0)+\sum_{\alpha=1}^{M_{1}} g\left(\lambda_{\alpha}^{1}\right)+\sum_{\alpha=1}^{\nu} g\left(\tilde{\lambda}_{\alpha}\right)
$$

Therefore,

$$
\frac{1}{N} \sum_{\alpha=1}^{M_{1}} g\left(\lambda_{\alpha}^{1}\right)=\int_{0}^{\Lambda_{I}} d \lambda \sigma(\lambda) g(\lambda)-\frac{1}{N} \sum_{\alpha=1}^{\nu} g\left(\tilde{\lambda}_{\alpha}\right)-\frac{1}{2 N} g(0)+\frac{1}{2 N} g\left(\Lambda_{I}\right) .
$$


Making the approximation $\Lambda_{I} \approx \infty$ introduces an error which is higher order in $1 / N$. We conclude that the sum (A.1) is given by

$$
\frac{1}{N} \sum_{\alpha=1}^{M_{1}} g\left(\lambda_{\alpha}^{1}\right)=\int_{0}^{\infty} d \lambda \sigma(\lambda) g(\lambda)-\frac{1}{N} \sum_{\alpha=1}^{\nu} g\left(\tilde{\lambda}_{\alpha}\right)-\frac{1}{2 N} g(0)
$$

(plus terms that are higher order in $1 / N)$. The presence of the last term $-\frac{1}{2 N} g(0)$ should be noted.

\section{References}

1. A.B. Zamolodchikov and Al.B. Zamolodchikov, Ann. Phys. 120 (1979) 253; A.B. Zamolodchikov, Sov. Sci. Rev. A2 (1980) 1.

2. P.P. Kulish and E.K. Sklyanin, J. Sov. Math. 19 (1982) 1596; Lecture Notes in Physics, Vol. 151 (Springer, 1982) 61; M. Jimbo, Int. J. Mod. Phys. A4 (1989) 3759.

3. I.V. Cherednik, Theor. Math. Phys. 61 (1984) 977.

4. E.K. Sklyanin, J. Phys. A21 (1988) 2375.

5. L. Mezincescu and R.I. Nepomechie, J. Phys. A24 (1991) L17.

6. L. Mezincescu, R.I. Nepomechie and V. Rittenberg, Phys. Lett. A147 (1990) 70; L. Mezincescu and R.I. Nepomechie, J. Phys. A25 (1992) 2533.

7. L. Mezincescu and R.I. Nepomechie, Int. J. Mod. Phys. A6 (1991) 5231; Addendum, A7 (1992) 5657.

8. L. Mezincescu and R.I. Nepomechie, Nucl. Phys. B372 (1992) 597.

9. C. Destri and H.J. de Vega, Nucl. Phys. B374 (1992) 692; B385 (1992) 361.

10. H.J. de Vega and A. González-Ruiz, J. Phys. A26 (1993) L519; preprints LPTHE-PAR 93-38; LPTHE-PAR 94-12

11. H.J. de Vega and A. González-Ruiz, preprint LPTHE-PAR 93-29

12. R.-H. Yue and Y.-X. Chen, J. Phys. A26 (1993) 2989; B.-Y. Hou and R.-H. Yue, Phys. Lett. A183 (1993) 169; R.-H. Yue, J. Phys. A27 (1994) 1633. 
13. M. Karowski and A. Zapletal, preprint (1993)

14. A. González-Ruiz, preprint F.T./U.C.M.-94/1

15. P.P. Kulish, in Proceedings of the Wigner Symposium, ed. V. Dobrev and H-D. Doebner

16. A. Fring and R. Köberle, preprints USP-IFQSC/TH/93-06; USP-IFQSC/TH/93-12; USP-IFQSC/TH/94-03

17. R. Sasaki, preprint YITP/U-93-33; E. Corrigan, P.Dorey, R.H. Rietdijk, R. Sasaki, preprint YITP/U-94-11.

18. S. Ghoshal and A. B. Zamolodchikov, preprint RU-93-20

19. S. Ghoshal, preprints RU-93-51; RU-94-02

20. L. Chim, preprint RU-94-33

21. F.C. Alcaraz, M.N. Barber, M.T. Batchelor, R.J. Baxter and G.R.W. Quispel, J. Phys. A20 (1987) 6397. See also M. Gaudin, Phys. Rev. A4 (1971) 386; La fonction d'onde de Bethe (Masson, 1983).

22. V.E. Korepin, Theor. Math. Phys. 76 (1980) 165; V.E. Korepin, G. Izergin and N.M. Bogoliubov, Quantum Inverse Scattering Method, Correlation Functions and Algebraic Bethe Ansatz (Cambridge University Press, 1993).

23. N. Andrei and C. Destri, Nucl. Phys. B231 (1984) 445.

24. P. Fendley and H. Saleur, preprint USC-94-001.

25. L.D. Faddeev and L.A. Takhtajan, J. Sov. Math. 24, 241 (1984).

26. C.J. Hamer, G.R.W. Quispel and M.T. Batchelor, J. Phys. A20 (1987) 5677. See also A.L. Owczarek and R.J. Baxter, J. Phys. A22 (1989) 1141; M.T. Batchelor and C.J. Hamer, J. Phys. A23 (1990) 761.

27. I. Affleck, preprint UBCTP-93-25

28. C.J. Hamer and M.T. Batchelor, J. Phys. A21 (1988) L173;

29. M. Takahashi, Prog. Theor. Phys. 46 (1971) 401. 
30. M. Gaudin, Phys. Rev. Lett. 26 (1971) 1301

31. A.M. Tsvelick and P.B. Wiegmann, Adv. in Phys. 32 (1983) 453.

32. R. Giles, L.D. McLerran, and C.B.Thorn, Phys. Rev. D17 (1978) 2058; F. Woynarovich and H.P. Eckle, J. Phys. A20 (1987) L97; M. Karowski, Nucl. Phys. B300 (1988) 473.

33. E.T. Whittaker and G.N. Watson, A Course of Modern Analysis (Cambridge University Press, 1988). 